\title{
Colour Image Processing
}

Joan Batlle, Lluís Pacheco,

Institute of Informatics and Applications

University of Girona. Technical School

Av. Lluís Santaló sn 17071 Girona, Spain

\{jbatlle,lluispa@eia.udg.edu\}

\begin{abstract}
In the context of he round table the following topics related to image colour processing will be discussed: Historical point of view. Studies of Aguilonius, Gerritsen, Newton and Maxwell. CIE Standard (Commission International de l'Eclaraige). Colour Models. RGB, HIS, etc. Colour segmentation based on HSI model. Industrial applications. Summary and discussion. At the end, video images showing the robustness of colour in front of $B / W$ images will be presented.
\end{abstract}

Index Terms - colour processing, colour models.

\section{INTRODUCTION}

Colour perception can be broken down in four stages:

- The light source.

- The "coloured" object.

- The eye with a colour-sensitive mechanism.

- The brain to interpret the energy messages received.

The human eye:

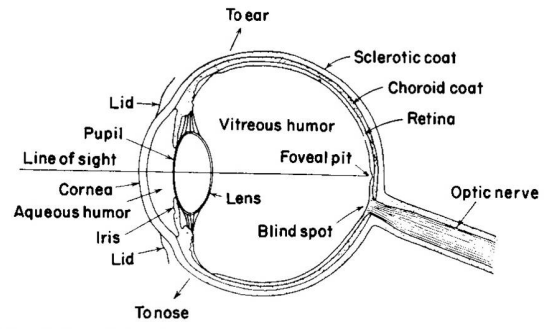

Fig. 1.1. A horizontal section through the human eye.

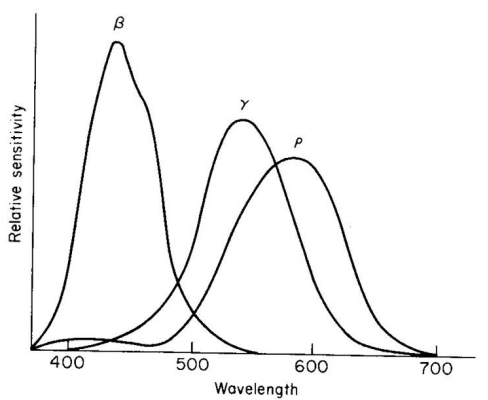

Fig. 1.2. Probable sensitivity curves of the colour receptors.

\section{HumAN AND SCIENTIFIC POINTS OF VIEW OF COLOUR INTERPRETATION TRHOUGHT THE HISTORY}

Aguilonius model - 1613

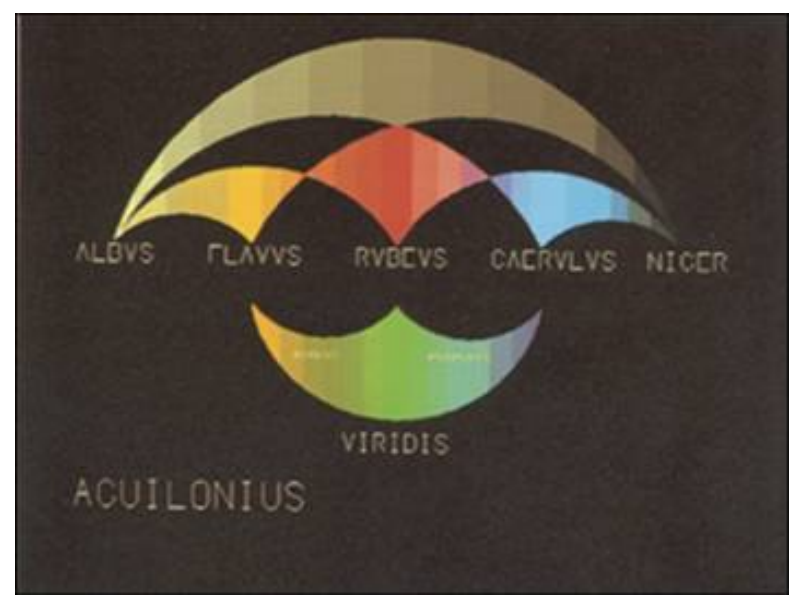

Newton's

experiment

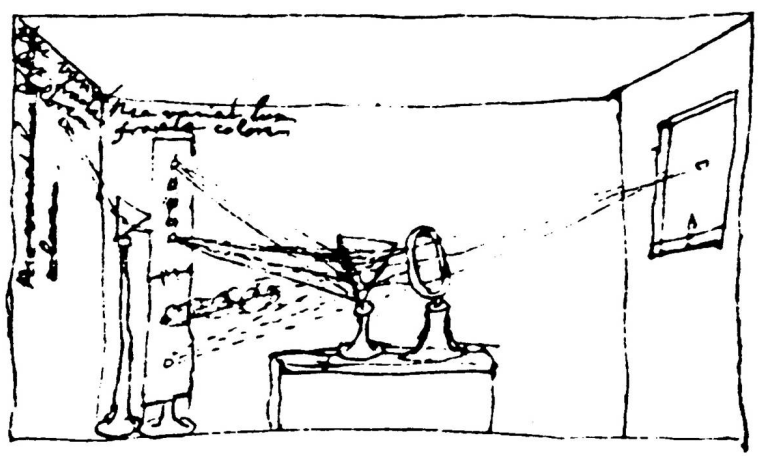

Frans Gerritsen adaptation (1988) of Newton's esperimental model (1660)

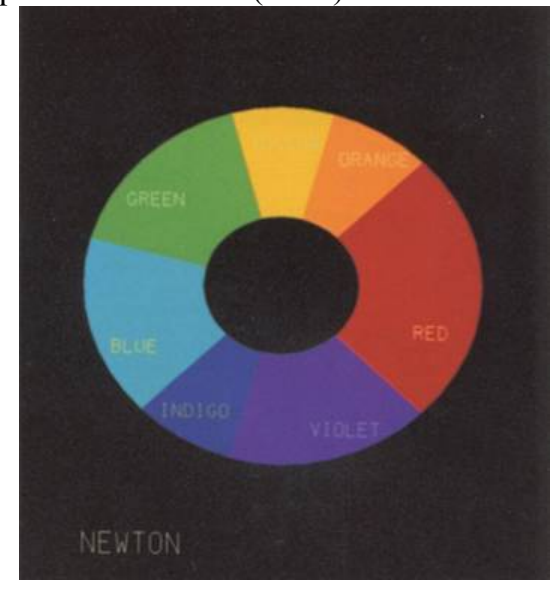

Goethe Triangle (1747-1832) 


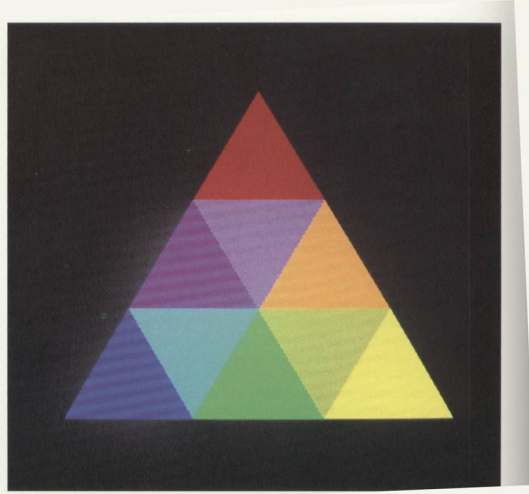

Maxwell Triangle (1872)

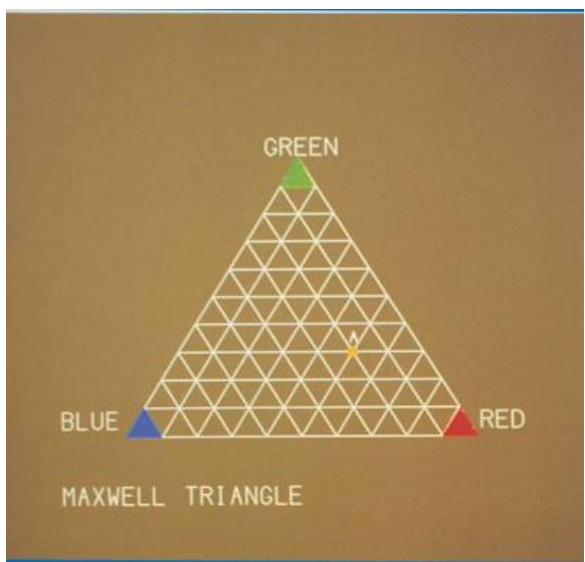

Modified Maxwell's Triangle (1747-1832)

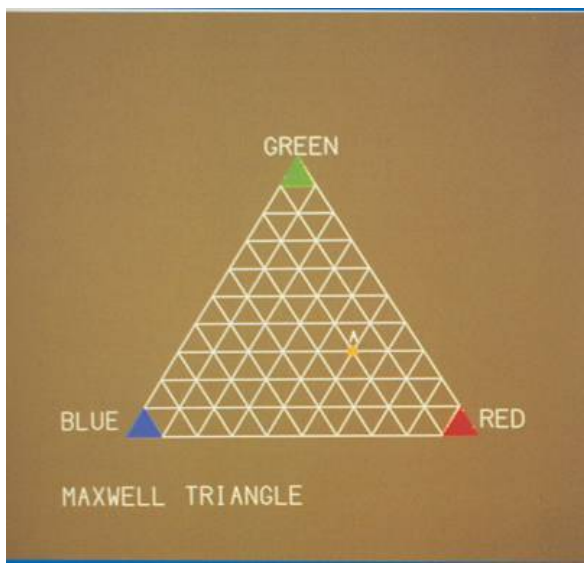

Model of Albert H.Munsell (1915)

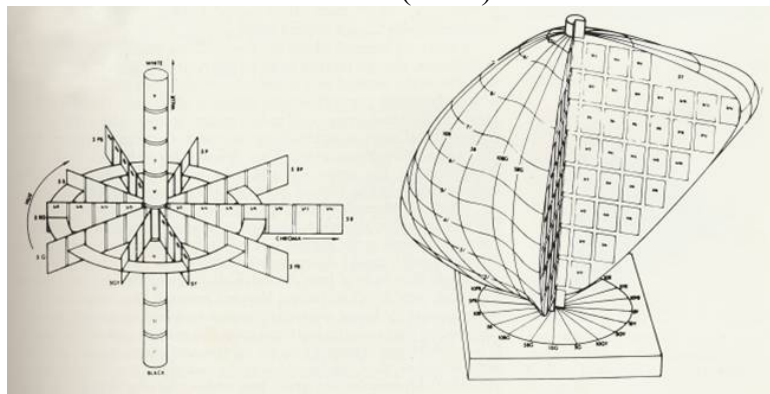

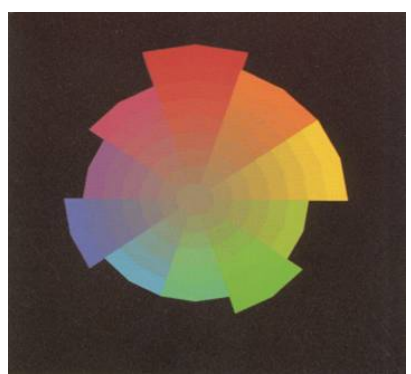

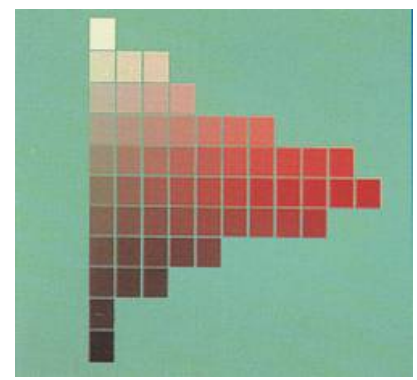

CIE - Commission International de l'Eclaraige (1931). Uniformed Space Colour (1976)
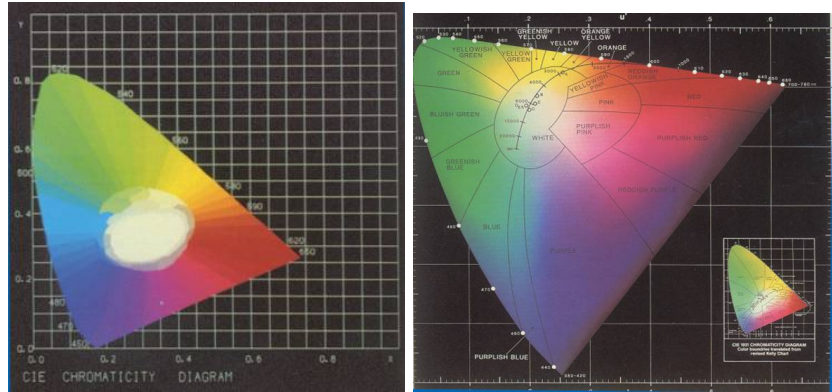

Judd Transformation (1931), adapted by CIE in 1960

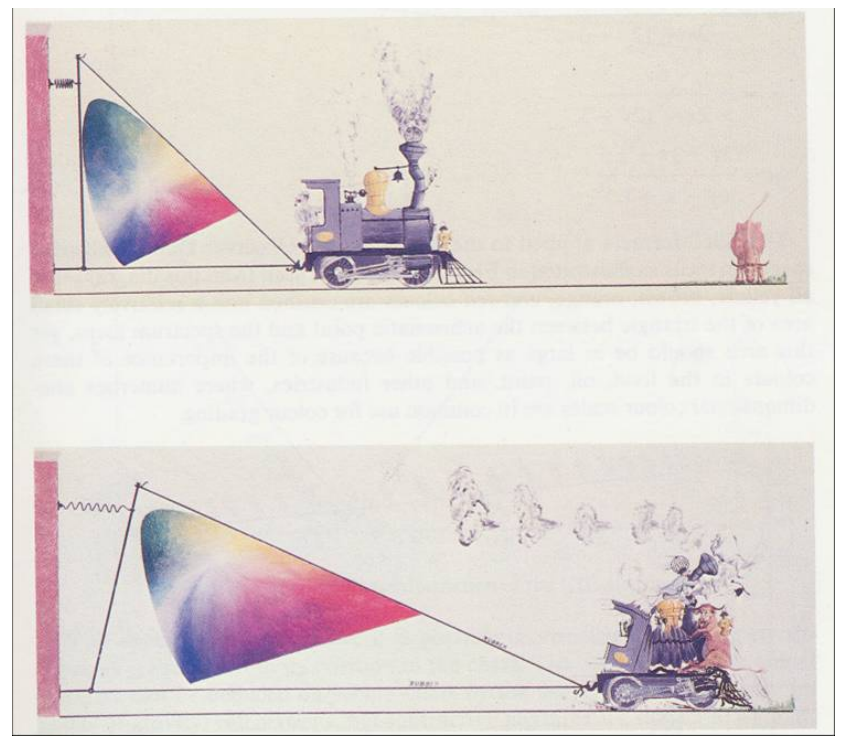

RGB Model of the CIE

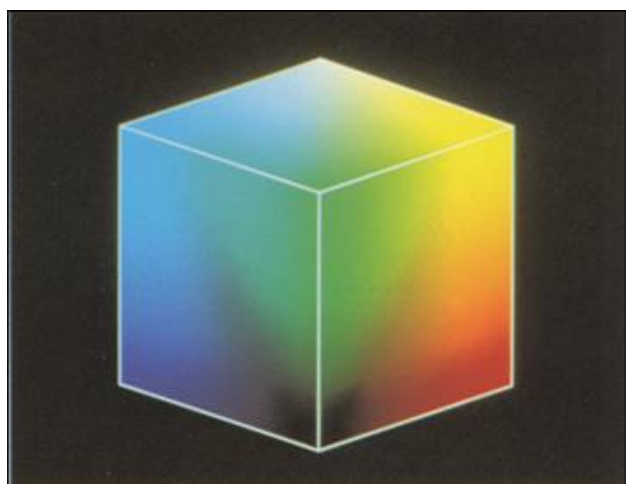




\section{Gerritsen Space (1975)}
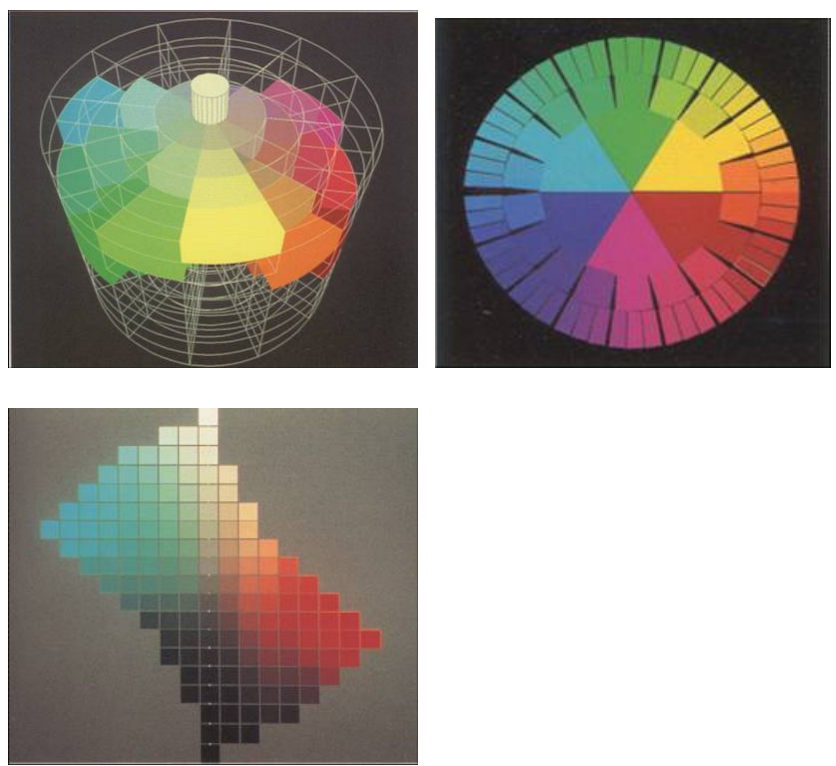

HSI Model
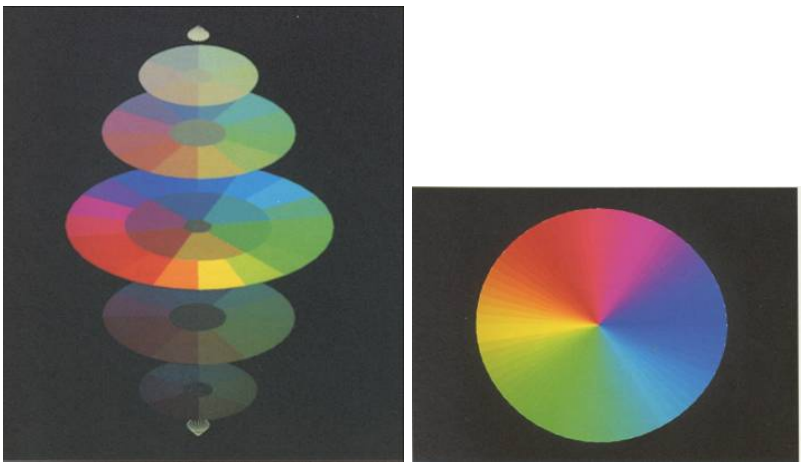

Hue: wave length

$H=\cos ^{-1}\left(\frac{1 / 2((R-G)+(R-B))}{\sqrt{(R-G)^{2}+(R-B)(G-B)}}\right)$

Saturation: colour purity

$S=1-\frac{\min (R, G, B)}{\frac{R+G+B}{3}}$

Intensity: amount of reflected light by one object

$I=(R+G+B) / 3$

\section{Human perception and eye calibration.}

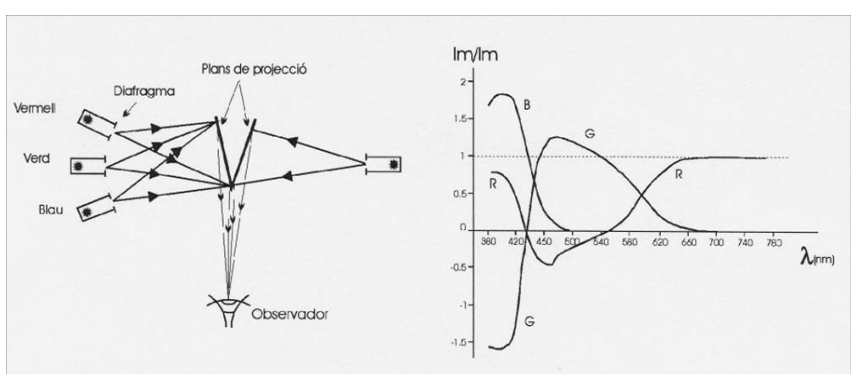

RGB versus HSI stability in front of illumination changes. Robustness of HUE.
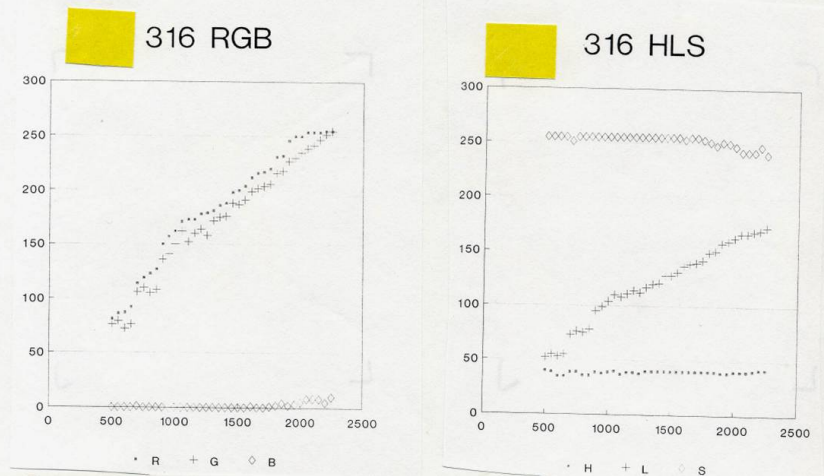

Invariants (vertical straight lines). Colour versus $B / W$ images.

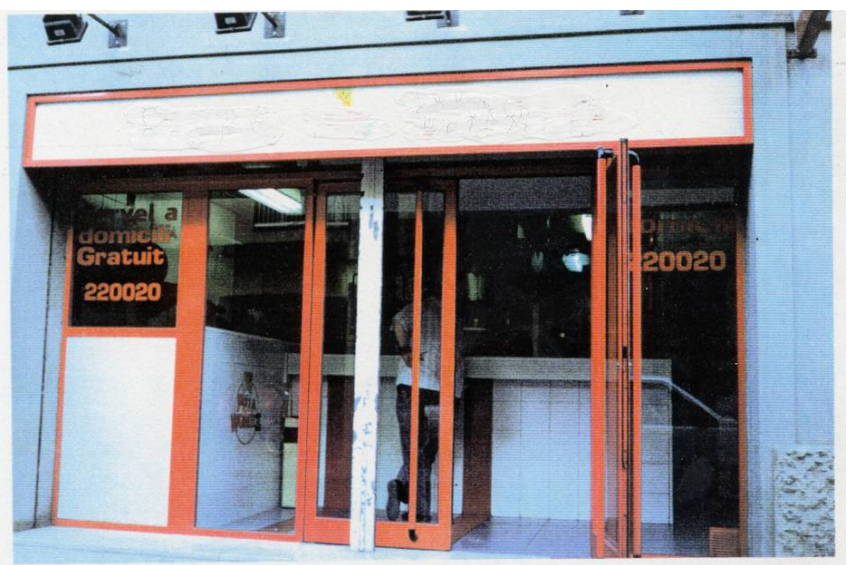

Extracted vertical straight lines from colour and $\mathrm{B} / \mathrm{W}$ information

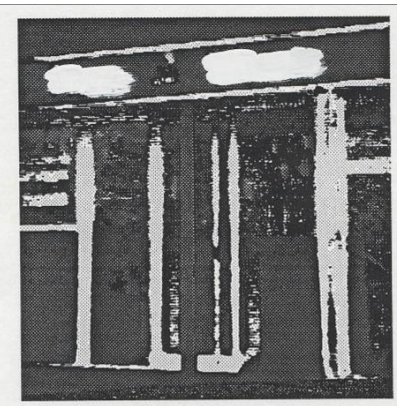

Fig-3.17 Hue

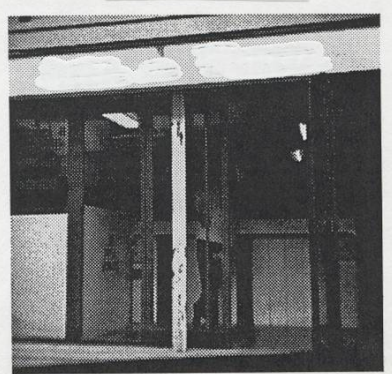

Fig-3.17 Lluminància

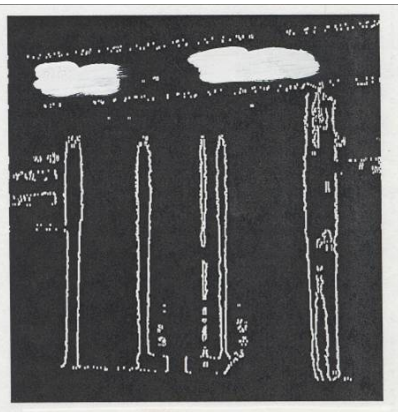

Fig-3.17 Rectes verticals sobre el Hue

表:

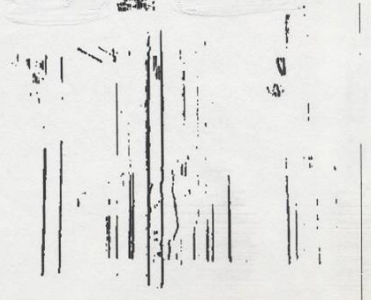

Fig-3.17 Rectes verticals sobre b Luminincia 
Robustness in front of illumination changes

500 Lux
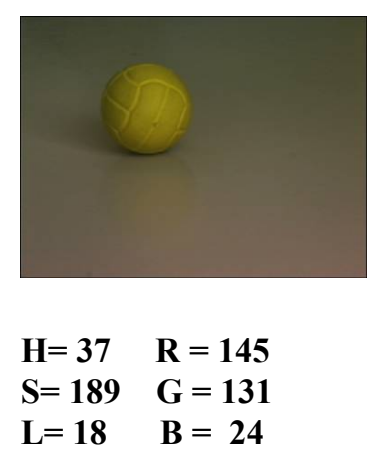

$H=33 \quad R=210$

$\mathrm{S}=199 \quad \mathrm{G}=190$

$\mathrm{L}=35 \quad B=35$

Real time segmentation using Hue component
Source image

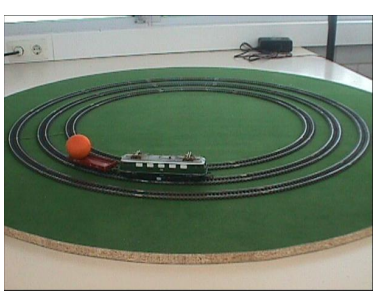

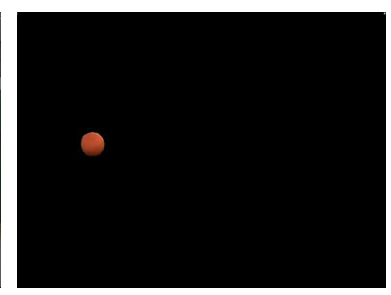

Segmented imag
III. COMMON COLOUR SPACES

R G B: $\quad \mathrm{R}$ or $\mathrm{R} /(\mathrm{R}+\mathrm{G}+\mathrm{B})$

$B$ or $B /(R+G+B)$

$G$ or $G /(R+G+B)$

HSI (Carron i Lambert):

$H=\left\{\begin{array}{l}\arccos \left(\frac{\frac{1}{2}((R-G)+(R-B))}{\sqrt{(R-G)^{2}+(R-B)(G-B)}}\right) \quad \text { if } B \leq G \\ 2 \Pi-\arccos \left(\frac{\frac{1}{2}((R-G)+(R-B))}{\sqrt{(R-G)^{2}+(R-B)(G-B)}}\right) \quad \text { if } B>G\end{array}\right.$

HSV (Smith):

$H=\left\{\begin{array}{l}60 *\left(b^{\prime}-g^{\prime}\right) \quad \text { if } R=\max (R, G, B) \\ 60 *\left(2+r^{\prime}-b^{\prime}\right) \text { if } G=\max (R, G, B) \\ 60 *\left(4+r^{\prime}-g^{\prime}\right) \text { if } B=\max (R, G, B)\end{array}\right.$

$S=\frac{\max (R, G, B)-\min (R, G, B)}{\max (R, G, B)}$

$V=\max (R, G, B)$

Where: $r^{\prime}=\frac{\max (R, G, B)-R}{\max (R, G, B)-\min (R, G, B)}$

$g^{\prime}=\frac{\max (R, G, B)-G}{\max (R, G, B)-\min (R, G, B)}$

$b^{\prime}=\frac{\max (R, G, B)-B}{\max (R, G, B)-\min (R, G, B)}$

HSV (Tenenbaum):

$H=\arctan \left(\frac{\sqrt{3}(G-B)}{2 R-G-B}\right) \quad V=\frac{R+G+B}{3}$

$S=1-3 * \min \left(\frac{R}{R+G+B}, \frac{G}{R+G+B}, \frac{B}{R+G+B}\right)$

Espai CIEL*a*b (Celenk):

$\mathrm{X}=2.769 \mathrm{R}-1.751 \mathrm{G}+1.130 \mathrm{~B}$

$\mathrm{Y}=1.000 \mathrm{R}-4.590 \mathrm{G}+0,060 \mathrm{~B}$

$Z=0.000 R-0.056 G+5.594 B$

$L^{*}=116\left[Y / Y_{0}\right]^{1 / 3}-16 \quad Y / Y_{0}>0.01$

$\left.a^{*}=500 \mathrm{~d}\left(X / X_{0}\right)^{1 / 3}-\left(Y / Y_{0}\right)^{1 / 3}\right] X / X_{0}>0.01$

$b^{*}=200\left[\left(Y / Y_{0}\right)^{1 / 3}-\left(Z / Z_{0}\right)^{1 / 3}\right] Z / Z_{0}>0.01$

$L^{*}=L^{*} \quad H^{\mathrm{o}}=\tan ^{-1}\left(b^{*} / a^{*}\right)$

$C^{*}=\left(a^{*^{2}}+b^{*^{2}}\right)^{1 / 2}$

OHTA Model:

$I_{1}=\frac{R+G+B}{3} \quad I_{2}=R-B \quad I_{3}=\frac{2 G-R-B}{2}$

\section{Joblove/Greenbegr's model}

This is a linear model almost the same as Smith one that presents a small modification on the saturation, the transformation you can see below:

$\mathrm{V}=(\max (\mathrm{R}, \mathrm{G}, \mathrm{B})+\min (\mathrm{R}, \mathrm{G}, \mathrm{B})) / 2$ 


\section{Tenenmaum's model}

This is a non linear model that presents almost the same bases as the HIS model complemented before (previous page). Its transformations are presented below:

$H=\arctan \frac{\sqrt{3}(G-B)}{(2 R-G-B)}$

$S=1-3 \min \left(\frac{R}{R+G+B}, \frac{G}{R+G+B}, \frac{B}{R+G+B}\right)$

$V=\frac{R+G+B}{3}$

\section{Yagi/Abe/Nakatani's model}

These authors present two similar models which origin/bases are in Smith's model

\section{Model 1: $\quad H=$ the same as Smith's model}

$$
\begin{aligned}
& S=\max (R, G, B)-\min (R, G, B) \\
& V=\frac{\max (R, G, B)+\min (R, G, B)}{2}
\end{aligned}
$$

Model 2: $\quad H=$ the same as Smith's model

$$
\begin{aligned}
& S=\max (R, G, B)-\min (R, G, B) \\
& V=\frac{R, G, B}{3}
\end{aligned}
$$

\section{VIDEO IMAGES}

Some results about colour applications in industrial environments will be presented. Real time segmented images will show how colour features can provided much more information than $\mathrm{B} / \mathrm{W}$ when colour is a feature of the scenario. Real time colour segmentation of natural environments will be also presented.
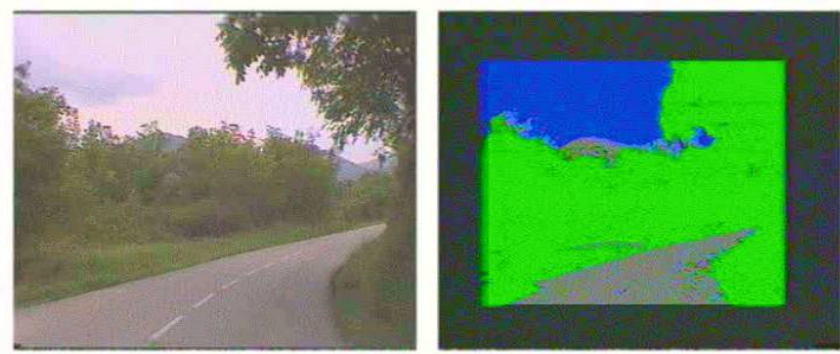

\section{REFERENCES}

[1] Richard B.Norman, "Electronic Color", Van Nostrand Reinhold. New York.
[2] Batlle, J.; Casals, A.; Freixenet, J.; Marti, J. “A review on strategies for recognizing natural objects in colour images of outdoor scenes" Image and Vision Computing, 2000

[3] Marti, J.; Freixenet, J.; Batlle, J.; Casals, A. "A new approach to outdoor scene description based on learning and top-down segmentation" Image and Vision Computing, 2001

[4] Lladó, X.; Martí, J.; Petrou, M. "Image texture prediction using colour photometric stereo" Lecture Notes in Artificial Intelligence, 2002

[5] Freixenet, J.; Muñoz, X.; Martí, J.; Lladó, X. "Color texture segmentation by region-boundary cooperation" Lecture Notes in Artificial Intelligence, 2004

[6] Garcia, R.; Batlle J,; Salvi, J. “A New Approach to Pose Detection using a Trinocular Stereovision System” Real-Time Imaging

[7] Muñoz, X.; Freixenet, J.; Cufí, X.; Martí, J. "Strategies for image segmentation combining region and boundary information" Pattern Recognition Letters , 2003

[8] Bosch, A.; Muñoz, X.; Martí, R. "Which is the best way to organize/classify images by content? A Review" Image and Vision Computing, 2007

[9] Bosch, A.; Muñoz, X. ; Freixenet, J. "Segmentation and descrition of natural outdoor scenes" Image and Vision Computing, 2007 